\title{
Circular RNA hsa_circ_0005909 modulates osteosarcoma progression via the miR-936/ HMGB1 axis
}

Shuai Ding, Guangquan Zhang ${ }^{*}$ (D, Yanzheng Gao, Shulian Chen and Chen Cao

\begin{abstract}
Background: Osteosarcoma (OS) is the most common bone malignant tumor in children, youth, and adolescents. Circular RNA hsa_circ_0005909 (circ_0005909) is involved in the progression of OS. Nevertheless, there are few reports on the role and mechanism of circ_0005909 in OS.

Methods: Quantitative real-time polymerase chain reaction (qRT-PCR) was executed to examine the expression of circ_0005909, miR-936, and High Mobility Group Box 1 (HMGB1) mRNA in OS tissues and cells. Cell viability, colony formation, migration, and invasion were evaluated by Cell Counting Kit-8 (CCK-8), cell colony formation, or transwell assays. Cell epithelial-mesenchymal transition (EMT) and HMGB1 protein levels were assessed through western blot analysis. The role of circ_0005909 on tumor growth in vivo was verified by xenograft assay. The relationship between circ_0005909 or HMGB1 and miR-936 was confirmed with the dual-luciferase reporter or RNA pull-down assays.

Results: Circ_0005909 level was upregulated in OS tissues and cells. OS patients with high circ_0005909 expression had a lower survival rate. Circ_0005909 inhibition reduced tumor growth in vivo and constrained cell viability, colony formation, migration, invasion, and EMT of OS cells in vitro. Furthermore, circ_0005909 served as a sponge for miR-936 and the repressive impacts of circ_0005909 silencing on malignant behaviors of OS cells were abolished by miR-936 inhibitors. Also, HMGB1 acted as a target for miR-936 and was modulated by circ_0005909 via miR-936. Additionally, HMGB1 overexpression restored the inhibitory influence on the malignant behaviors of OS cells mediated by circ_0005909 inhibition.
\end{abstract}

Conclusions: Circ_0005909 inhibition impeded the progression of OS via downregulating HMGB1 via sponging miR-936.

Keywords: OS, circ_0005909, miR-936, HMGB1, OS cell lines

*Correspondence: zgqdoctor@163.com

Department of Spine and Spinal Cord, Henan Provincial People's Hospital, People's Hospital of Zhengzhou University, People's Hospital of Henan University, No. 7, Weiwu Road, Jinshui District, Zhengzhou 450003, Henan, China

\section{Highlights}

1. The expression of circ_0005909 was elevated in OS tissues and cells.

2. Inhibition of circ_0005909 decreased the malignant behaviors of OS cells.

3. Circ_0005909 acted as a sponge for miR-936 in OS cells.

4. HMGB1 served as a target for miR-936 in OS cells.

5. Circ_0005909 regulated HMGB1 expression through sponging miR-936.

(c) The Author(s) 2020. This article is licensed under a Creative Commons Attribution 4.0 International License, which permits use, sharing, adaptation, distribution and reproduction in any medium or format, as long as you give appropriate credit to the original author(s) and the source, provide a link to the Creative Commons licence, and indicate if changes were made. The images or other third party material in this article are included in the article's Creative Commons licence, unless indicated otherwise in a credit line to the material. If material is not included in the article's Creative Commons licence and your intended use is not permitted by statutory regulation or exceeds the permitted use, you will need to obtain permission directly from the copyright holder. To view a copy of this licence, visit http://creativeco mmons.org/licenses/by/4.0/. The Creative Commons Public Domain Dedication waiver (http://creativecommons.org/publicdomain/ zero/1.0/) applies to the data made available in this article, unless otherwise stated in a credit line to the data. 


\section{Background}

Osteosarcoma (OS) is a primary bone tumor that originates from mesenchymal cells and is common in children, youth, and adolescents $[1,2]$. The malignant disease is characterized by rapid progress, early metastasis, and high mortality [3]. At present, the combination of surgical resection and systemic multi-drug chemotherapy is the main method for OS treatment and the long-term overall survival rate for patients with OS is approximately 65-70\% [4]. However, the overall 5-year survival rate of patients with metastasis and recurrence of OS was about $30 \%$ and $15 \%$, respectively [5]. In consequence, exploring the molecular mechanism of OS advancement is indispensable for improving the prognosis of patients with OS.

Circular RNAs (circRNAs), a new type of endogenous non-coding RNAs, have a closed circular structure formed by covalent attachment [6]. Recently, circRNAs have been shown to interact with RNA-binding proteins and act as splicing and transcription regulators and microRNA (miRNA) sponges [7]. Also, circRNAs were associated with the advancement of tumors $[8,9]$. Circular RNA hsa_circ_0005909 (circ_0005909) is derived from the reverse splicing of XPR1 mRNA and has a length of 371 nucleotides. Circ_0005909 was revealed to promote the expression of CADM1 in OS cells via acting as a sponge for miRNAs [10]. At present, the role and mechanisms of circ_0005909 in OS are rarely reported.

MiRNAs are another type of non-coding RNAs that can regulate the expression of targeted genes at the posttranscriptional level [11]. They are implicated in almost every aspects of cancer biologies, such as angiogenesis, invasion and metastasis, apoptosis, and proliferation [12]. MiR-936 was connected with the progression of epithelial ovarian cancer [13], glioma [14], and non-small cell lung cancer [15]. However, the role of miR-936 in OS has not been fully elucidated.

High Mobility Group Box 1 (HMGB1) has a dual function, it can act as a DNA chaperone inside the cells, and it works with chemokines, cytokines, and growth factors outside the cells [16]. It was reported that HMGB1 played an important role in many diseases, including cancers [17]. The genetic variation HMGB1 gene might serve as a predictor for breast cancer advancement and metastasis [18]. HMGB1 was disclosed to be connected with the progression of a variety of cancers [19-22]. Moreover, HMGB1 was involved in the sensitivity of OS to drugs and the apoptosis and proliferation of OS cells [23]. However, it is unclear whether HMGB1 can be regulated by circ_0005909 and miR-936 in OS.

Hence, we verified the role of circ_0005909 in OS. Furthermore, we also investigated the mechanism of the circ_0005909/miR-936/HMGB1 axis in OS.

\section{Materials and methods OS specimens}

54 patients with OS underwent surgical treatment at Henan Provincial People's Hospital, and their tumors tissues and neighboring non-tumor tissues were used in this study. The inclusion criteria were as follows: preoperatively confirmed as osteosarcoma by puncture or biopsy; no immunotherapy, chemotherapy, radiotherapy, or chemotherapy before surgery; patients agreed to participate in this study and clinical data were complete. The exclusion criteria were as follows: suffering from other malignant tumors; suffering from other types of serious diseases; patients received treatment for other clinical diseases within 3 months before admission; pregnant or breastfeeding. All patients informed consents before undergoing surgical resection. The association between circ_0005909 expression and clinicopathological parameters of OS patients were presented in Table 1. This research was ratified by the Ethics Committee of Henan Provincial People's Hospital.

\section{Cell culture and transfection}

Normal osteoblast hFOB1.19 and OS cell lines (HOS, $\mathrm{U} 2 \mathrm{OS}$, and SAOS-2) were purchased from the American Type Culture Collection (Manassas, VA, USA). All cells were kept in an incubator with $5 \% \mathrm{CO}_{2}$ at $37{ }^{\circ} \mathrm{C}$ and cultured in Dulbecco's modified Eagle's medium (DMEM, Life Technologies, Grand Island, NY, USA)

Table 1 Correlation between circ_0005909 expression and clinicopathological parameters of patients with OS

\begin{tabular}{|c|c|c|c|c|}
\hline \multirow[t]{3}{*}{ Characteristics } & \multirow[t]{3}{*}{ Number } & \multicolumn{2}{|c|}{$\begin{array}{l}\text { circ_0005909 } \\
\text { expression }\end{array}$} & \multirow[t]{3}{*}{$P$} \\
\hline & & High & Low & \\
\hline & & 27 & 27 & \\
\hline Age (years) & & & & 0.586 \\
\hline$<18$ & 28 & 15 & 13 & \\
\hline$\geq 18$ & 26 & 12 & 14 & \\
\hline Gender & & & & 0.783 \\
\hline Male & 31 & 16 & 15 & \\
\hline Female & 23 & 11 & 12 & \\
\hline Tumor size (cm) & & & & 0.301 \\
\hline$\leq 5$ & 33 & 16 & 17 & \\
\hline$>5$ & 21 & 11 & 10 & \\
\hline Clinical staging & & & & $0.029^{*}$ \\
\hline$|-| \mid$ & 28 & 10 & 18 & \\
\hline III & 26 & 17 & 9 & \\
\hline Distant metastasis & & & & $0.014^{*}$ \\
\hline Present & 25 & 17 & 8 & \\
\hline Absent & 29 & 10 & 19 & \\
\hline
\end{tabular}

${ }^{*} P<0.05$, statistically significant 
complemented with fetal bovine serum (FBS, 10\%, Sigma, Louis, Missouri, MO, USA), streptomycin $(100 \mu \mathrm{g} / \mathrm{mL}$, Sigma), and penicillin (100 U/mL, Sigma).

Short hairpin RNA targeting circ_0005909 (sh-circ\#1 and sh-circ\#2) and negative control (sh-NC) were bought from RiboBio (Guangzhou, China). MiRNA mimics and inhibitors targeting miR-936 (miR-936 and anti-miR-936) and their negative controls (miR-NC and anti-NC) were procured from RiboBio. The sequence of HMGB1 was cloned into the pcDNA3.1 vector (vector) (Invitrogen, Carlsbad, CA, USA) to construct the overexpression vectors for HMGB1. When the cells were cultured to approximately $80 \%$ confluence, OS cells (HOS and U2OS) were transiently transfected with the assigned oligonucleotides or vectors using Lipofectamine 3000 reagent (Invitrogen) and cultured for $48 \mathrm{~h}$.

\section{RNA preparation, RNase $\mathrm{R}$ digestion, and quantitative} real-time polymerase chain reaction (qRT-PCR)

The nuclear and cytoplasmic RNA of OS cells were isolated with the PARIS KIT50 RXNS (Life Technologies). Total RNA was extracted through the TRIzol reagent (Invitrogen). For RNase R digestion, total RNA of OS cells was treated with RNase R (3 U/ $\mu$ g, Epicentre Technologies, Madison, WI, USA) at $37^{\circ} \mathrm{C}$ for $15 \mathrm{~min}$. Total RNA $(5 \mu \mathrm{g})$ was reversely transcribed into complementary DNA using the PrimeScript RT reagent Kit (Takara, Dalian, China) or miRNA First-Strand Synthesis Kit (Takara). QRT-PCR was conducted through the SYBR Premix Ex Taq (Takara). The expression of circ_0005909, XPR1, miR-936, and HMGB1 was figured by the $2^{-\Delta \Delta C t}$ method. The primers used in the research were displayed as below: Glyceraldehyde-3-phosphate dehydrogenase (GAPDH): (Forward: 5'-GACTCCACTCAC GGCAAATTCA-3' and Reverse: $5^{\prime}$-TCGCTCCTGGAA GATGGTGAT-3'); circ_0005909: (Forward: $5^{\prime}$-GTATCC ACTTGCCCTTTA-3' and Reverse: 5'-TTACTCCAG CCTGTCTC-3'); XPR1: (Forward: 5'-TCCACCTAC GGAGGACAATC-3' and Reverse: 5'-GGAGAAGTG CAGGCAAGAAC-3'); HMGB1 (Forward: 5'-GGGATG GCAAAGTTTTTCCCTTTA-3' and Reverse: 5'-CAC TAACCCTGCTGTTCGCT-3'); U6 small nuclear RNA (snRNA) (Forward: 5'-GCTCGCTTCGGCAGCACA $-3^{\prime}$ and Reverse: 5'-GAGGTATTCGCACCAGAGGA-3') and miR-936: (Forward: 5'-AACGAGACGACGACA GAC-3' and Reverse: 5'-ACAGTAGAGGGAGGAATC GCAG-3'). GAPDH or U6 snRNA was served as an internal control.

\section{Cell Counting Kit-8 (CCK-8) assay}

The assigned vectors or oligonucleotides were transfected into OS cells for $48 \mathrm{~h}$. After washing with phosphate buffer solution (PBS), the cells were incubated with DMEM mixed the CCK-8 reagent $(10 \mu \mathrm{L}$, Dojindo, Tokyo, Japan) for $2 \mathrm{~h}$. The absorbance at $450 \mathrm{~nm}$ was measured through the Microplate Absorbance Reader (Bio-Rad Labs., Richmond, CA, USA).

\section{Cell colony formation assay}

The transfected HOS and U2OS cells $\left(1.5 \times 10^{2}\right.$ cells/well $)$ were seeded in 6-well plates and maintained at $37{ }^{\circ} \mathrm{C}$ in an incubator with $5 \% \mathrm{CO}_{2}$ for 2 weeks, and the medium was replaced every 4 days. After fixation with formaldehyde (10\%, Sigma) for $20 \mathrm{~min}$, cell colonies in each well were stained with crystal violet $(0.1 \%$, KeyGen, Jiangsu, China) for $5 \mathrm{~min}$. The colonies (>50 cells) were counted and photographed under a light microscope (Olympus, Tokyo, Japan).

\section{Transwell assay}

After transfection for $48 \mathrm{~h}$, the HOS and U2OS cells were collected. In the invasion assay, the transfected HOS and U2OS cells $\left(1 \times 10^{5}\right)$ were seeded on the upper chamber of the transwell chamber $(8 \mu \mathrm{m}$, Corning Costar, Corning, NY, USA) with matrigel matrix (BD Biosciences, San Jose, CA, USA). And the lower chamber of the transwell chamber was supplemented with the culture medium with FBS (10\%). The cells on the upper membrane were removed after culturing for $24 \mathrm{~h}$. Subsequently, the cells on the lower membrane were fixed with paraformaldehyde (4\%, Beyotime, Shanghai, China) and stained with crystal violet $(0.1 \%$, KeyGen). The same procedures were used for the migration assay, but the upper chamber of the transwell chamber was not covered with the matrigel matrix. The migrated and invaded cells were figured with a light microscope (Olympus).

\section{Western blot analysis}

Western blot analysis was executed as previously described [24]. OS tissues and cells were lysed in lysis buffer (Beyotime). After the subjection of the sodium dodecyl sulfate polyacrylamide gel electrophoresis (SDSPAGE), the total protein was transferred onto the polyvinylidene difluoride (PVDF) membranes (Invitrogen). The membranes were probed with anti-N-cadherin (N-cad) (ab18203, 1:1000, Abcam, Cambridge, MA, USA), antiE-cadherin (E-cad) (ab40772, 1:10,000, Abcam), antiHMGB1 (ab79823, 1:500, Abcam), and anti-GAPDH (ab128915, 1:5000, Abcam), and then incubated with goat anti-rabbit (ab97051, 1:10,000, Abcam) immunoglobulin G (IgG). GAPDH was regarded as a loading control. Protein bands were visualized by the ImmunoStar LD (Wako Pure Chemical, Osaka, Japan). 


\section{Xenograft assay}

The animal experiment was ratified by the Ethics Committee of Henan Provincial People's Hospital. 8 BALB/c nude mice (athymic, 4-5 weeks old) were purchased from Charles River Laboratories (Beijing, China) and were fed under specific-pathogen-free conductions. HOS cells $\left(1 \times 10^{6}\right)$ with sh-NC or stable lentivirus-mediated sh-circ_0005909 (sh-circ\#1, RiboBio) were subcutaneously injected into the dorsal side of the nude mice (4 mice/group). The tumor volume was measured once a week using the equation: Volume $=\left(\right.$ length $\times$ width $\left.^{2}\right) / 2$. The mice were euthanized on day 35 after injection for subsequent analysis.

\section{Dual-luciferase reporter assay}

The binding sites of miR-936 in circ_0005909 were predicted by the Circinteractome database. The sequences of circ_0005909 and mutant circ_0005909 (within the binding sites to miR-936) was inserted into the pGL3-control vector (Promega, Madison, WI, USA) for the construction of the wild type (WT) and mutant (MUT) luciferase reporter vectors for hsa_circ_0000517, respectively. The binding sites of HMGB1 in miR-936 were predicted by the Targetscan database. The same method was employed to construct the luciferase reporter vectors for HMGB1. HOS and U2OS cells were cotransfected luciferase reporter vectors and miR-NC or miR-936. The dualluciferase reporter assay kit (Promega) was employed to assess the luciferase intensities of the luciferase reporter vectors in OS cells.

\section{RNA pull-down assay}

In brief, the Biotinylated (Bio)-miR-NC and Bio-miR-936 from Sigma were transfected into OS cells, respectively. The lysates of OS were incubated with Dynabeads M-280 Streptavidin (Invitrogen) at $4{ }^{\circ} \mathrm{C}$ for $3 \mathrm{~h}$ to pull down the Bio-coupled RNA complexes. Following this, the beads were washed through the lysis buffer (Beyotime), and the complex was purified by TRIzol (Invitrogen). The enrichment of circ_0005909 in the complex was examined through qRT-PCR.

\section{Statistical analysis}

The experiments of the research in vitro were repeated at the last 3 times. GraphPad Prism 7.0 (GraphPad, La Jolla, CA, USA) and SPSS 18.0 software (SPSS, Chicago, IL, USA) were utilized for statistical analysis. The association between circ_0005909 expression and clinicopathological parameters of patients with OS was analyzed with the Chi square test. The survival curve was assessed via the Kaplan-Meier method and the log-rank test. The differences between the OS tissues and neighboring nontumor tissues were analyzed by using paired Student's $t$ test. The differences between the two groups were determined by unpaired Student's $t$ test, and the differences among more groups were analyzed with one-way analysis of variance (ANOVA) followed by Tukey post hoc test. The correlation was evaluated via Pearson's correlation analysis. Differences were deemed significant if $P<0.05$. Data were exhibited as mean \pm standard deviation.

\section{Results \\ Expression and characteristic of circ_0005909 in OS}

At first, we examined the expression pattern of circ_0005909 in 54 paired OS tissues and neighboring non-tumor tissues through qRT-PCR. We observed that circ_0005909 expression was overtly increased in OS tissues with respect to that in the neighboring non-tumor tissues (Fig. 1a). Consistently, a distinct augmentation of circ_0005909 was observed in OS cell lines (HOS, U2OS, and SAOS-2) compared to the hFOB1.19 cells (Fig. 1b). Moreover, circ_0005909 expression was associated with clinical staging and distant metastasis (Table 1). Furthermore, patients with OS were divided into two groups based on the median value: high circ_0005909 expression group and low circ_0005909 expression group, and the overall survival of OS patients with the high expression of circ_0005909 were lower than the OS patients with low circ_0005909 expression (Fig. 1c). Also, compared to the liner gene XPR1, circ_0005909 was resistant to RNase R (Fig. 1d, e). Besides, we detected the relative expression of circ_0005909 in the cytoplasm and nucleus of HOS and U2OS cells. QRT-PCR displayed that circ_0005909 was primary located in the cytoplasm of HOS and U2OS cells (Fig. 1f). These findings revealed that circ_0005909 was abundant and stable in OS tissues and cells, and high circ_0005909 expression might be associated with OS advancement.

\section{Circ_0005909 silencing constrained viability, colony formation, migration, invasion, and epithelial-mesenchymal transition (EMT) of OS cells in vitro and repressed $O S$ growth in vivo}

Considering that circ_0005909 was upregulated in OS tissues and cells, we further surveyed the role of circ_0005909 in OS through loss-function-experiments. In contrast to the control sh-NC, circ_0005909 expression was drastically reduced in $\mathrm{HOS}$ and U2OS cells transfected with sh-circ\#1 and sh-circ\#2, while the expression of XPR1 mRNA did not change (Fig. 2a). Also, the expression of circ_0005909 was lower in the sh-circ\#1 group with respect to the sh-circ\#2 group, so we employed the sh-circ\#1 to explore the impacts of circ_0005909 silencing on viability, colony formation, migration, and invasion of OS cells. CCK- 8 assay revealed that decreased circ_0005909 expression impeded cell 

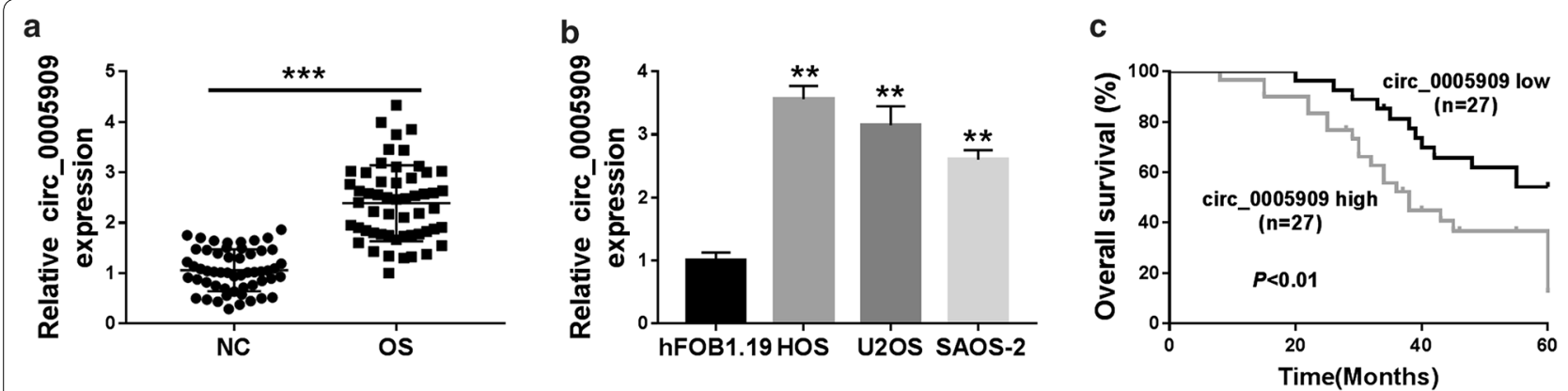

d

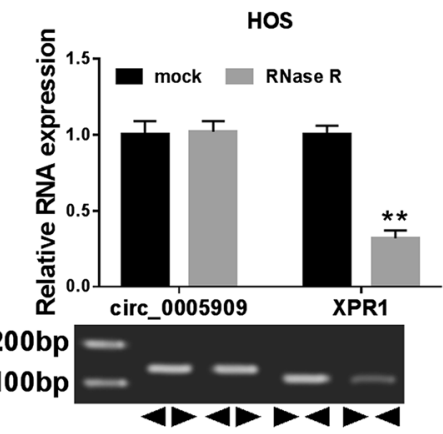

e

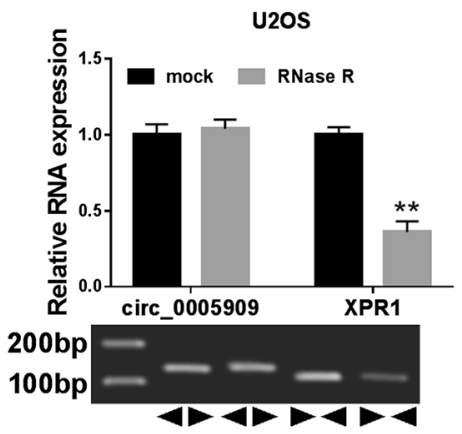

f

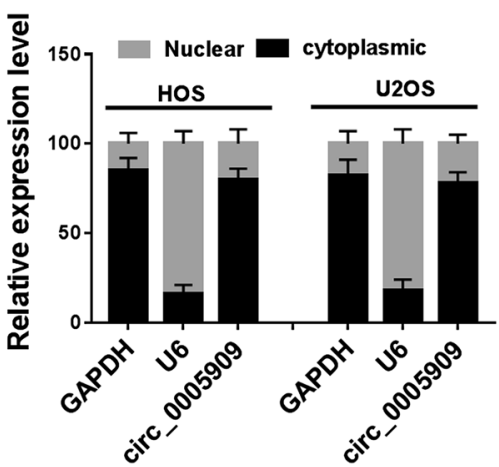

Fig. 1 Circ_0005909 expression was enhanced in OS tissues and cells. a, b Expression of circ_0005909 in OS tissues (a) and cells (b) was analyzed through qRT-PCR. c The overall survival of OS patients with a high circ_0005909 expression and low circ_0005909 expression was determined via Kaplan-Meier's curves. d, e The abundance of circ_0005909 and XPR1 in HOS and U2OS cells treated with RNase R was analyzed by qRT-PCR.f The abundance of circ_0005909 in the cytoplasm or nucleus of HOS and U2OS cells was assessed with qRT-PCR. ${ }^{* *} P<0.01$ and ${ }^{* * *} P<0.001$

viability in HOS and U2OS cells (Fig. 2b). Cell colony formation assay exhibited that the colony formation ability of HOS and U2OS cells was inhibited by circ_0005909 downregulation (Fig. 2c). Transwell assay displayed that circ_0005909 reduction evidently curbed cell migration and invasion capacities in HOS and U2OS cells (Fig. 2d, e). Moreover, the EMT associated proteins, E-cad and $\mathrm{N}$-cad, were detected via western blot analysis in circ_0005909-silenced HOS and U2OS cells. The results exhibited that E-cad protein expression was upregulated and $\mathrm{N}$-cad protein expression was downregulated in circ_0005909-silenced HOS and U2OS cells (Fig. 2f). Subsequently, we explored the role of circ_0005909 in vivo through xenograft models. The results presented that tumor volume and weight of mice in the sh-circ\#1 group were remarkably curbed compared to the control group (Fig. 2g, h). Collectively, these results indicated that circ_0005909 knockdown repressed OS cell growth in vitro and in vivo.

\section{Circ_ 0005909 acted as a sponge for miR-936 in OS cells}

To investigate the molecular mechanism of circ_0005909 in OS, we predicted the target for circ_0005909 through the Circinteractome database. We discovered that 8
miRNAs (miR-338-3p, miR-411, miR-498, miR-515-5p, miR-561, miR-605, miR-766, and miR-936) had possible binding sites for circ_0005909. Silenced circ_0005909 expression could elevate the expression of miR-338-3p and miR-936 in HOS and U2OS cells, while there was no distinct difference in the other miRNAs (Additional file 1: Fig. S1). It was reported that circ_0005909 acted as a sponge for miR-338-3p in OS cells [10], so we chose miR-936 for subsequent researches. The potential binding sites between circ_0005909 and miR-936 were shown in Fig. 3a. After miR-936 mimics transfection, miR-936 expression was overtly increased in HOS and U2OS cells compared to the control group (Additional file 2: Fig S2A). Moreover, dual-luciferase reporter assay exhibited that miR-936 overexpression obviously suppressed the luciferase intensity of the circ_0005909-WT reporter vectors in $\mathrm{HOS}$ and U2OS cells, while the luciferase activity of the circ_0005909-MUT reporter vectors did not change (Fig. 3b, c). Also, RNA pull-down assay manifested that circ_0005909 was enriched in HOS and U2OS cells with Bio-miR-936 compared to the control group (Fig. 3d). Moreover, miR-936 expression was elevated in circ_0005909-inhibited HOS and U2OS cells (Fig. 3e). Furthermore, miR-936 expression was effectively reduced 


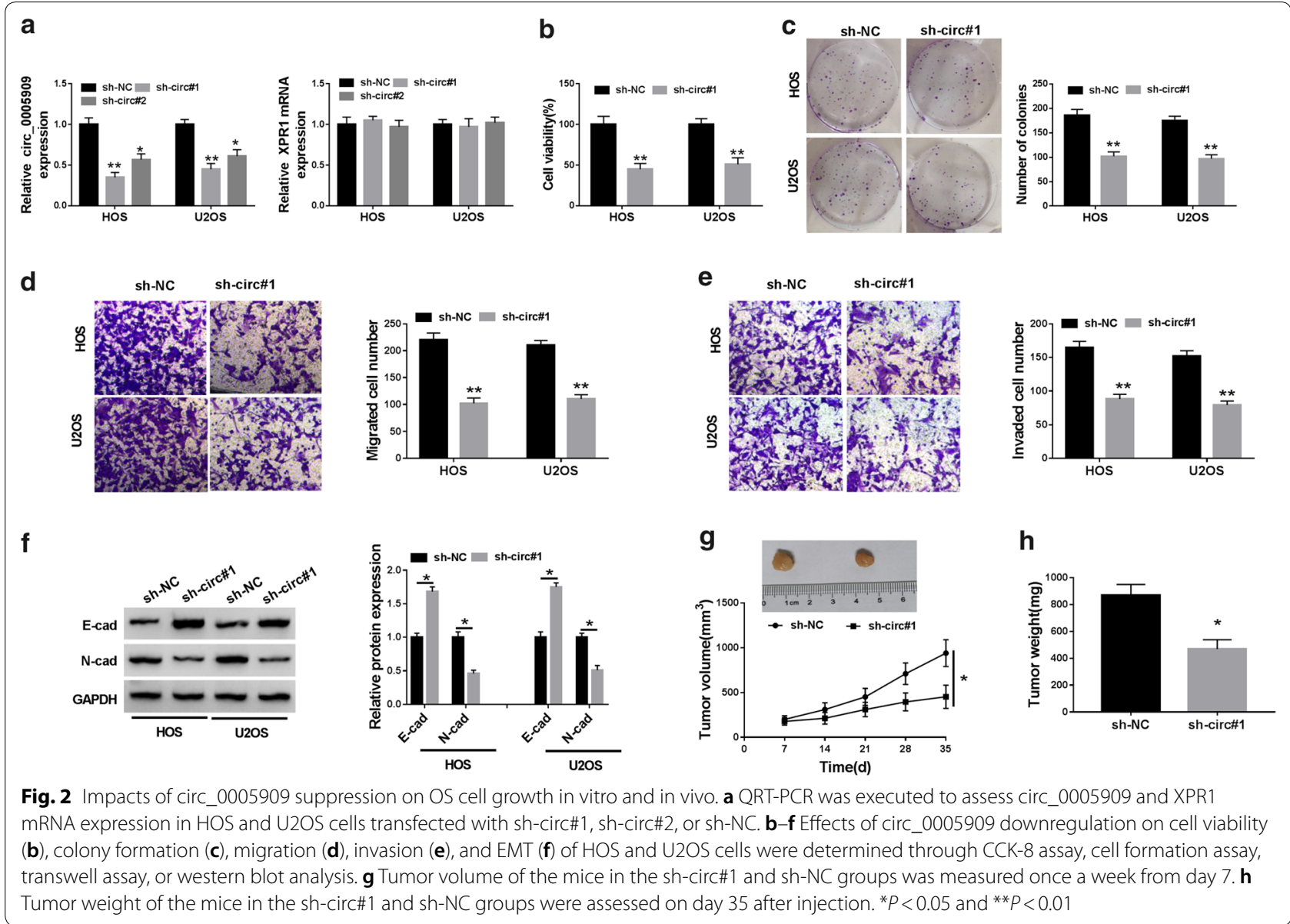

in HOS, U2OS, and SAOS-2 cells compared with the hFOB1.19 cells (Fig. 3f). The same expression trend of miR-936 was observed in OS tissues (Fig. 3g). Additionally, circ_0005909 and miR-936 had a negative correlation in OS tissues (Fig. 3h). We also explored the influence of miR-936 overexpression on viability, colony formation, migration, and invasion of OS cells. The results exhibited that miR-936 upregulation decreased cell viability, colony formation, migration, and invasion of U2OS and SAOS-2 cells (Additional file 2: Fig S2B-S2E). Together, these data indicated that circ_0005909 acted as a sponging for miR936 in OS cells.

\section{MiR-936 suppression abolished circ_0005909} silencing-mediated effects on cell viability, colony formation, migration, invasion, and EMT in OS cells Given that miR-936 served as a target for circ_0005909 in OS cells, we explored whether circ_0005909 played its role via miR-936. In comparison to the anti-NC, the expression of miR-936 was curbed in HOS and U2OS cells transfected with anti-miR-936 (Fig. 4a). Moreover, the repression of viability and colony formation of
HOS and U2OS cells mediated by circ_0005909 silencing was abrogated by miR-936 inhibitors (Fig. 4b, c). Also, the repressive impacts of circ_0005909 inhibition on migration and invasion of HOS and U2OS cells were overturned by miR-936 downregulation (Fig. 4d, e). Besides, silenced miR-936 expression reversed the effects of circ_0005909 inhibition on the levels of N-cad and E-cad proteins in HOS and U2OS cells (Fig. 4f, g). Taken together, these results demonstrated that circ_0005909 mediated cell viability, colony formation, migration, invasion, and EMT in OS cells via miR-936.

\section{HMGB1 was a target for miR-936 in OS cells}

Subsequently, we further surveyed the downstream target for miR-936 via Targetscan database. Online bioinformatics prediction (TargetScan) exhibited that 8 genes (E2F3, GSK3B, MAPK8, TWIST1, HMGB1, wmt5a, ATM, and IGF1) might be downstream targets of miR-936. Furthermore, miR-936 mimics could reduce the level of HMGB1 mRNA in HOS and U2OS cells, so that HMGB1 was selected for subsequent studies (Additional file 3: Fig. S3). As exhibited in Fig. 5a, 


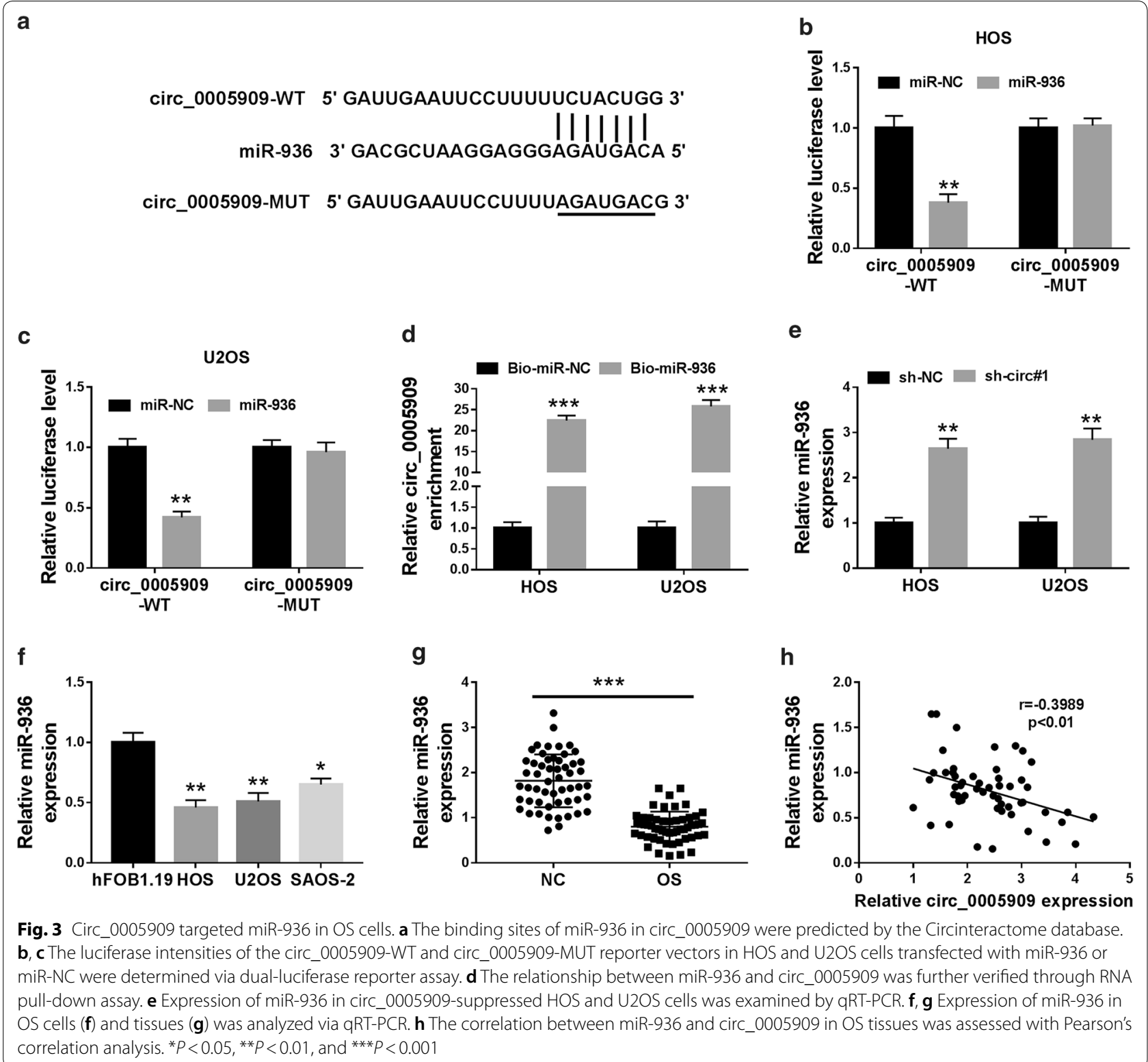

the $3^{\prime} \mathrm{UTR}$ of HMGB1 had latent binding sites for miR936. Dual-luciferase reporter assay suggested that the luciferase activity of the luciferase reporter vectors with HMGB1 $3^{\prime}$ UTR-WT was reduced in HOS and U2OS cells transfected with miR-936, while there was no overt difference in the luciferase reporter vectors containing HMGB1 3'UTR-MUT (Fig. 5b, c). Elevated miR-936 expression reduced HMGB1 mRNA and protein levels in HOS and U2OS cells (Fig. 5d, e). Also, HMGB1 mRNA and protein levels were decreased in circ_0005909-inhibited HOS and U2OS cells, while this influence was restored by miR-936 downregulation
(Fig. 5f, g). Therefore, these findings disclosed that HMGB1 acted as a target for miR-936 in OS cells.

\section{HMGB1 elevation restored circ_0005909}

downregulation-mediated influence on viability, colony formation, migration, invasion, and EMT of OS cells Knowing that circ_0005909 regulated HMGB1 expression via miR-936 in OS cells, we further surveyed whether circ_0005909 modulated cell viability, colony formation, migration, invasion, and EMT in OS cells via HMGB1. Compared to the control vectors, HMGB1 protein level was evidently increased in HOS and U2OS cells after HMGB1 transfection (Fig. 6a). Furthermore, 


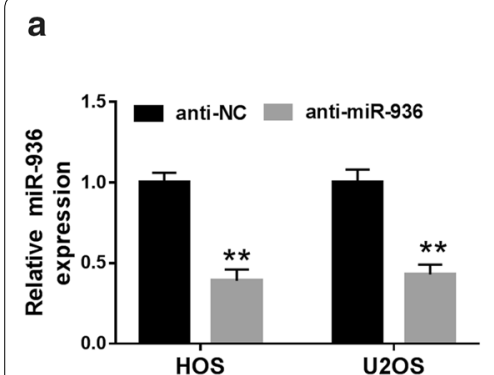

d

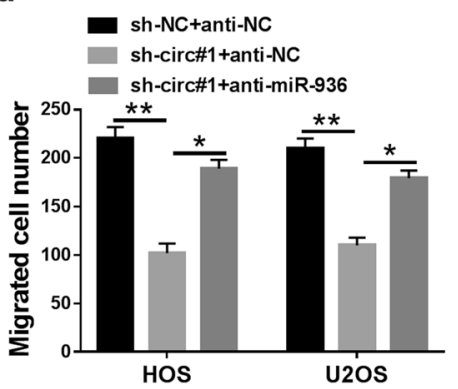

g

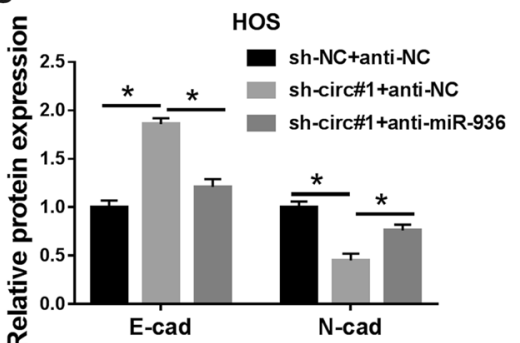

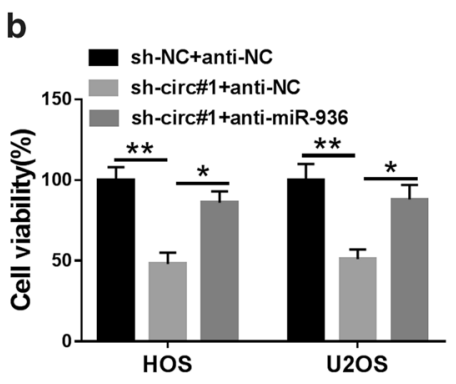
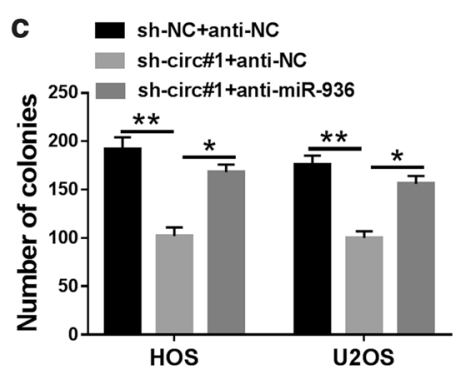

e

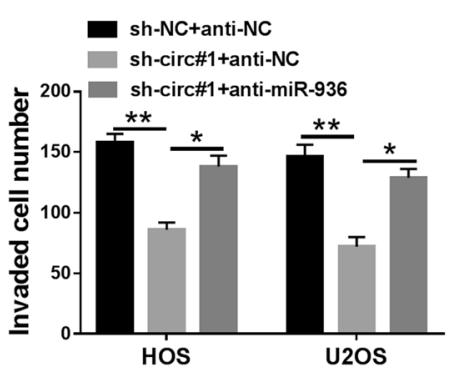

f

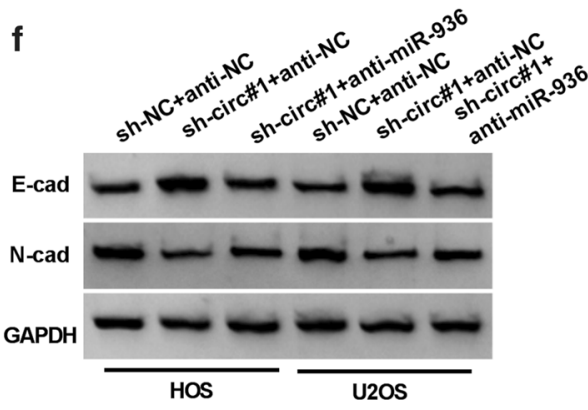

Fig. 4 Circ_0005909 regulated cell malignant behaviors in OS cells via miR-936. a Expression of miR-936 in HOS and U2OS cells transfected with anti-miR-936 or anti-NC was determined with qRT-PCR. b-g Impacts of miR-936 inhibition on viability, colony formation, migration, invasion, and EMT of HOS and U2OS cells induced by circ_0005909 depletion were evaluated by CCK-8 assay (b), cell formation assay (c), transwell assay (d, e), or western blot analysis ( $\mathrm{F}$ and $\mathrm{G}) .{ }^{*} P<0.05$ and ${ }^{* *} P<0.01$

HMGB1 elevation recovered the inhibitory impacts of circ_0005909 silencing on viability and colony formation of HOS and U2OS cells (Fig. 6b, c). Moreover, forced HMGB1 expression overturned the suppressive influence on migration and invasion of HOS and U2OS cells caused by circ_0005909 inhibition (Fig. 6d, e). Additionally, both the upregulation of E-cad and the downregulation of N-cad in circ_0005909-blocked HOS and U2OS cells were reversed by HMGB1 overexpression (Fig. 6f, g). Therefore, these results suggested that circ_0005909 modulated cell malignant behaviors in OS cells via HMGB1.

\section{Discussion}

Accumulating researches have disclosed that circRNAs exert significant roles in different biological processes, particularly in the genesis, progression, and metastasis of tumors [25]. Also, circRNAs might be acted as promising biomarkers and targets for the diagnosis and treatment of tumors [26]. Recently, a series of researches demonstrated the importance of circRNA-mediated regulatory mechanisms on OS. For example, circRNA_100876 silencing inhibited OS progression by sponging miR136 [27]. CircRNA circTADA2A facilitated OS metastasis and development by increasing CREB3 expression via sponging miR-203a-3p [28]. Furthermore, circRNA circ_0001785 mediated HOXB2 expression via competitively binding to miR-1200 in OS cells, which could mediate the pathogenesis of OS [29]. In this study, we revealed that circ_0005909 silencing decreased tumor growth in vivo and curbed cell viability, colony formation, migration, invasion, and EMT in OS cells in vitro. Chen et al. [10] unmasked that circ_0005909 contributed to OS progression through elevating CADM1 expression via sponging miR-338-3p. These data manifested that circ_0005909 played a promotive role in OS progression. 


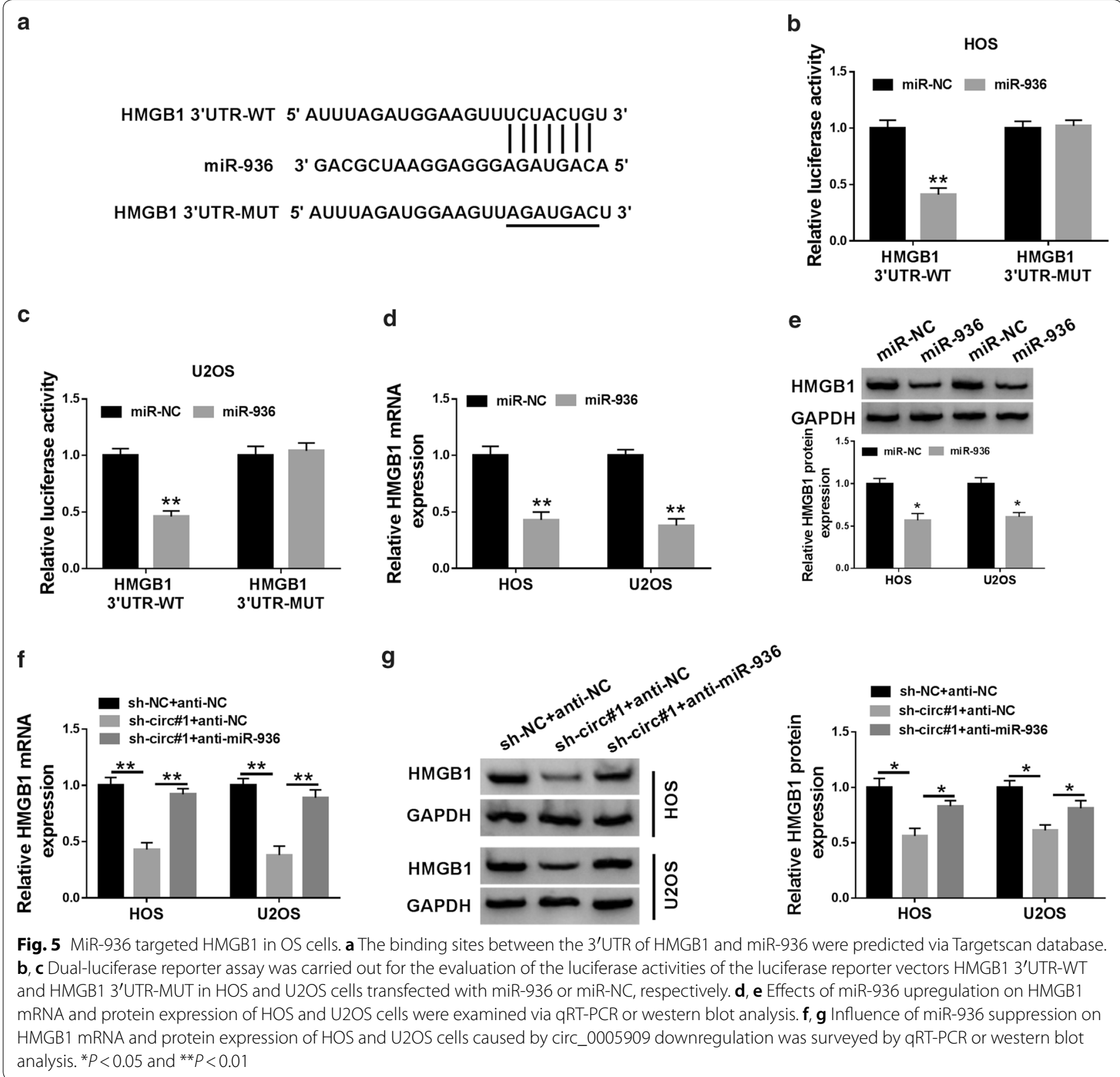

Given that circ_0005909 could act as a sponge for miR338-3p in OS [10], we disocovered that circ_0005909 severed as a sponge for miR-936 in OS cells. MiR-936 was revealed to be downregulated in several cancers. Zhou et al. [15] demonstrated that miR-936 upregulation impeded E2F2 expression in non-small cell lung cancer cells, which could constrain cell cycle progression, invasion, and proliferation of non-small cell lung cancer cells. Another report indicated that miR-936 suppressed the tumor aggressiveness by inactivating the PI3K/AKT pathway via repressing FGF2 expression in epithelial ovarian cancer [13]. Furthermore, miR-936 impeded gliomas progression by negatively regulating CKS1 expression and the AKT/ERK1/2 signaling pathway [14]. Herein, miR-936 inhibition reversed circ_0005909 blockingmediated the repressive effects on the malignant behaviors of OS cells. Therefore, we inferred that circ_0005909 modulated OS progression via sponging miR-936.

Subsequently, we surveyed the downstream targets of miR-936. We found that HMGB1 acted as a target for miR-936. Previous research revealed that HMGB1 was downregulated by lncRNA MALAT1 silencing via miR-129-5p or miR-142-3p, which could induce apoptosis and curbed the growth of OS cells [30]. Moreover, 


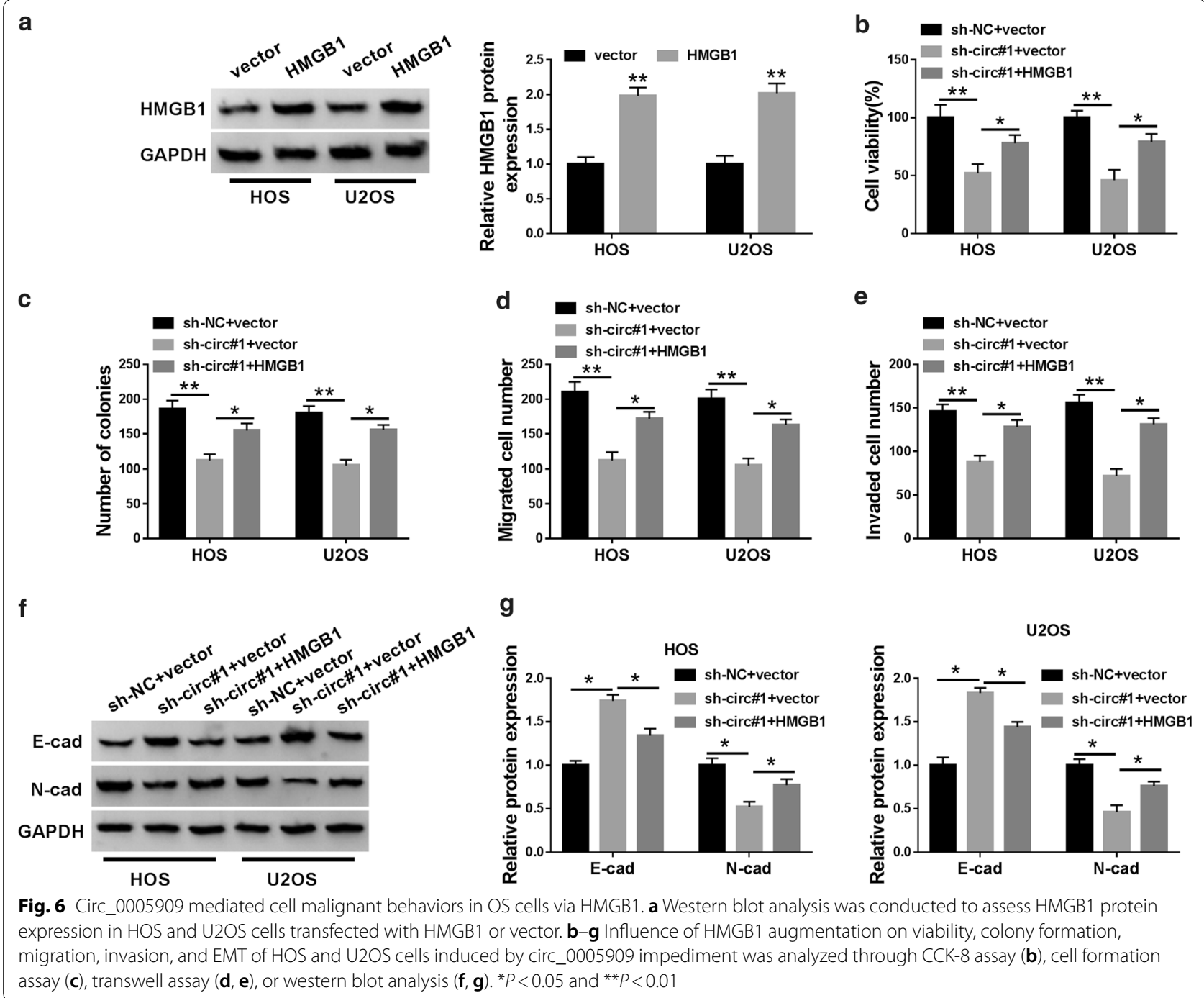

Liu et al. [31] claimed that miR-505 repressed cell invasion, migration, and proliferation in OS cells via downregulating HMGB1. Also, HMGB1 silencing could reduce the resistance of OS cells to drugs [32]. We revealed that HMGB1 was modulated by circ_0005909 via miR-936. Besides, HMGB1 overexpression abolished the repressive influence of circ_0005909 silencing on the malignant behaviors of OS cells. From all the above evidence, we concluded that circ_0005909 regulated the malignant behaviors of OS cells via regulating HMGB1 expression via sponging miR-936 (Fig. 7). Additionally, in the current study, we only explored the mechanism of circ_0005909/miR-936/ HMGB1 axis in OS. In the future, some signaling pathways are involved in the mechanism of circ_0005909/ miR-936/HMGB1 axis and whether circ_0005909 has the ability of protein translation can be studied in depth. 


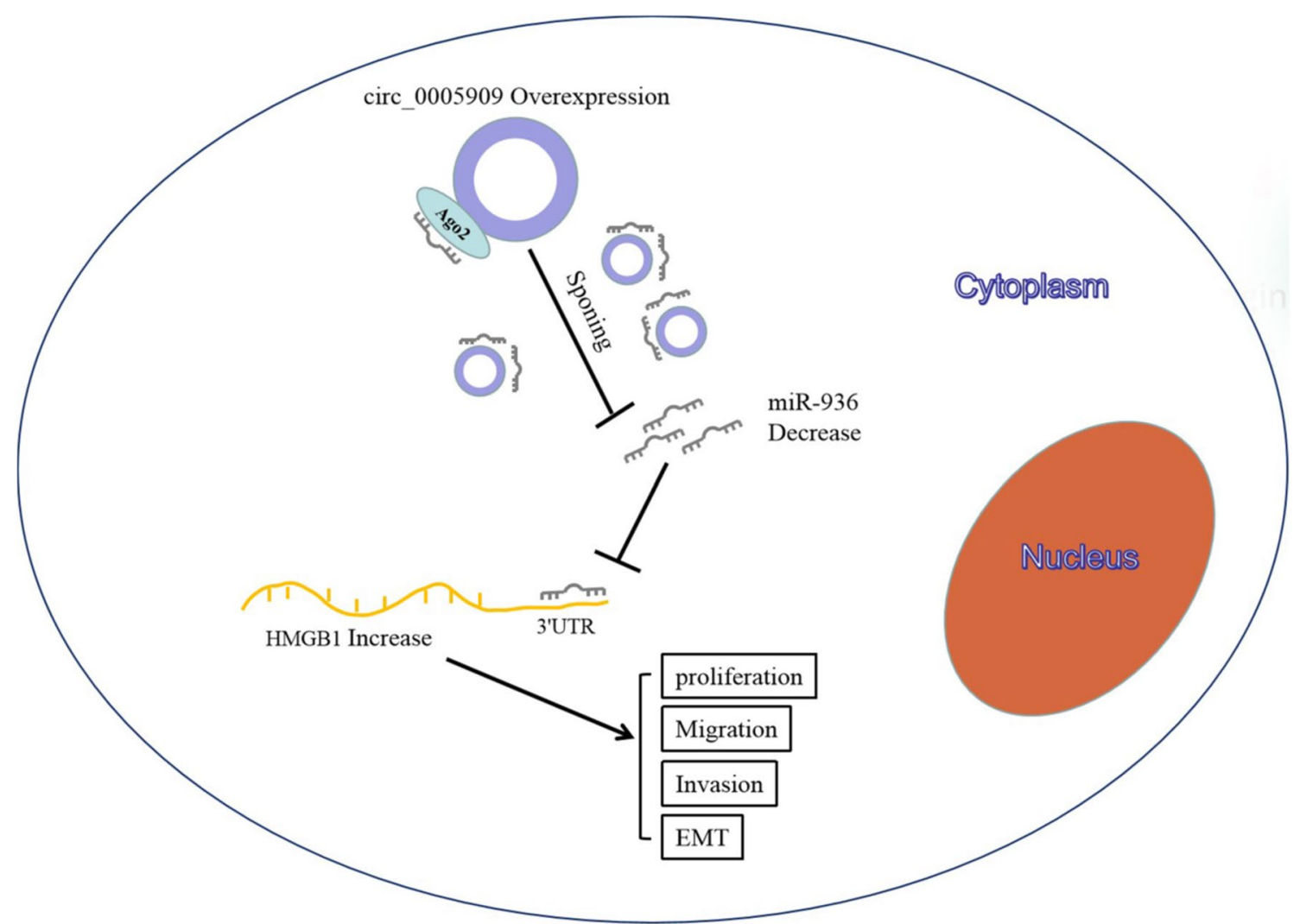

Fig. 7 The schematic cartoon of the circ_0005909/miR-936/HMGB1 axis in OS. Circ_0005909 overexpression can sponge more endogenous miR-936 to sequester and inhibit miR-936 activity, thereby leading to the upregulation of HMGB1, which facilitating the progression of OS

\section{Conclusion}

In all, circ_0005909 mediated the progression of OS via the miR-936/HMGB1 axis, which disclosed that circ_0005909 might be an underlying therapeutic target for OS patients.

\section{Supplementary information}

Supplementary information accompanies this paper at https://doi. org/10.1186/s12935-020-01399-1.

Additional file 1: Fig. S1. Effect of circ_0005909 suppression on the expression of miRNAs. (A and B) QRT-PCR was executed to assess the levels of miR-338-3p, miR-411, miR-498, miR-515-5p, miR-561, miR-605, miR-766, and miR-936 in HOS and U2OS cells transfected with shcirc_0005909\#1 or sh-NC. ${ }^{*} P<0.01$.

Additional file 2: Fig S2. Influence of miR-936 mimics on the malignant behaviors of OS cells. (A) After miR-936 or miR-NC transfection, the expression levels of miR-936 in HOS and U2OS cells were examined with qRT-PCR. (B-E) After miR-936 or miR-NC transfection, the viability, colony formation, migration, and invasion of $\mathrm{HOS}$ and U2OS cells were determined by CCK-8, cell formation, or transwell assays. ${ }^{*} P<0.01$ and ***P $<0.001$.

Additional file 3: Fig. S3. Impact of miR-936 mimics on the expression of its putative target genes. ( $A$ and $B$ ) QRT-PCR presented the mRNA levels of E2F3, GSK3B, MAPK8, TWIST1, HMGB1, wmt5a, ATM, and IGF1 in HOS and U2OS cells transfected with miR-936 mimics or miR-NC. ${ }^{*} P<0.01$.

\section{Abbreviations}

OS: Osteosarcoma; QRT-PCR: Quantitative real-time polymerase chain reaction; HMGB1: High Mobility Group Box 1; CCK-8: Cell Counting Kit-8; EMT: Epithelialmesenchymal transition; WT: Wild type.

\section{Acknowledgements}

Not applicable.

\section{Authors' contributions}

All authors made substantial contribution to conception and design, acquisition of the data, or analysis and interpretation of the data; take part in drafting the article or revising it critically for important intellectual content; gave final approval of the revision to be published; and agree to be accountable for all aspect of the work. All authors read and approved the final manuscript.

\section{Funding}

No funding was received.

\section{Availability of data and materials}

The analyzed data sets generated during the present study are available from the corresponding author on reasonable request.

\section{Ethics approval and consent to participate}

The present study was approved by the ethical review committee of the Henan Provincial People's Hospital.

\section{Patient consent for publication}

Not applicable. 


\section{Competing interests}

The authors declare that they have no competing interests.

Received: 9 March 2020 Accepted: 3 July 2020

Published online: 13 July 2020

\section{References}

1. Mirabello L, Troisi RJ, Savage SA. International osteosarcoma incidence patterns in children and adolescents, middle ages and elderly persons. Int J Cancer. 2009;125(1):229-34.

2. Sampson VB, Kamara DF, Kolb EA. Xenograft and genetically engineered mouse model systems of osteosarcoma and Ewing's sarcoma: tumor models for cancer drug discovery. Expert Opin Drug Discov. 2013;8(10):1181-9.

3. Sampo M, Koivikko M, Taskinen M, Kallio P, Kivioja A, Tarkkanen M, Böhling T. Incidence, epidemiology and treatment results of osteosarcoma in Finland-a nationwide population-based study. Acta Oncol. 2011;50(8):1206-14.

4. Isakoff MS, Bielack SS, Meltzer P, Gorlick R. Osteosarcoma: current treatment and a collaborative pathway to success. J Clin Oncol. 2015;33(27):3029-35.

5. Anninga JK, Gelderblom H, Fiocco M, Kroep JR, Taminiau AHM, Hogendoorn PCW, Egeler RM. Chemotherapeutic adjuvant treatment for osteosarcoma: where do we stand? Eur J Cancer. 2011;47(16):2431-45.

6. Memczak S, Jens M, Elefsinioti A, Torti F, Krueger J, Rybak A, Maier L, Mackowiak SD, Gregersen LH, Munschauer M, et al. Circular RNAs are a large class of animal RNAs with regulatory potency. Nature. 2013;495(7441):333-8.

7. Yin Y, Long J, He Q, Li Y, Liao Y, He P, Zhu W. Emerging roles of circRNA in formation and progression of cancer. J Cancer. 2019;10(21):5015-21.

8. Kristensen LS, Hansen TB, Venø MT, Kjems J. Circular RNAs in cancer: opportunities and challenges in the field. Oncogene. 2018;37(5):555-65

9. Patop IL, Kadener S. circRNAs in cancer. Curr Opin Genet Dev. 2018:48:121-7.

10. Chen G, Wang Q, Yang Q, Li Z, Du Z, Ren M, Zhao H, Song Y, Zhang G. Circular RNAs hsa_circ_0032462, hsa_circ_0028173, hsa_circ_0005909 are predicted to promote CADM1 expression by functioning as miRNAs sponge in human osteosarcoma. PLoS ONE. 2018;13(8):e0202896.

11. Kwan JYY, Psarianos P, Bruce JP, Yip KW, Liu F-F. The complexity of microRNAs in human cancer. J Radiat Res. 2016;57(Suppl 1):i106-11.

12. Lee YS, Dutta A. MicroRNAs in cancer. Annu Rev Pathol. 2009;4:199-227.

13. Li C, Yu S, Wu S, Ni Y, Pan Z. MicroRNA-936 targets FGF2 to inhibit epithelial ovarian cancer aggressiveness by deactivating the PI3K/Akt pathway. OncoTargets Ther. 2019;12:5311-22.

14. Wang D, Zhi T, Xu X, Bao Z, Fan L, Li Z, Ji J, Liu N. MicroRNA-936 induces cell cycle arrest and inhibits glioma cell proliferation by targeting CKS1. Am J Cancer Res. 2017;7(11):2131.

15. Zhou X, Tao H. Overexpression of microRNA-936 suppresses non-small cell lung cancer cell proliferation and invasion via targeting E2F2. Exp Ther Med. 2018;16(3):2696-702.

16. Kang R, Zhang Q, Zeh HJ, Lotze MT, Tang D. HMGB1 in cancer: good, bad, or both? Clin Cancer Res. 2013;19(15):4046-57.

17. Sims GP, Rowe DC, Rietdijk ST, Herbst R, Coyle AJ. HMGB1 and RAGE in inflammation and cancer. Annu Rev Immunol. 2010;28:367-88.
18. Huang B-F, Tzeng H-E, Chen P-C, Wang C-Q, Su C-M, Wang Y, Hu G-N, Zhao Y-M, Wang Q, Tang C-H. genetic polymorphisms are biomarkers for the development and progression of breast cancer. Int J Med Sci. 2018;15(6):580-6.

19. Gao H, Gong N, Ma Z, Miao X, Chen J, Cao Y, Zhang G. LncRNA ZEB2-AS1 promotes pancreatic cancer cell growth and invasion through regulating the miR-204/HMGB1 axis. Int J Biol Macromol. 2018;1 16:545-51.

20. Wang S, Chen Y, Yu X, Lu Y, Wang H, Wu F, Teng L. miR-129-5p attenuates cell proliferation and epithelial mesenchymal transition via HMGB1 in gastric cancer. Pathol Res Pract. 2019;215(4):676-82.

21. Qian F, Xiao J, Gai L, Zhu J. HMGB1-RAGE signaling facilitates Ras-dependent Yap1 expression to drive colorectal cancer stemness and development. Mol Carcinogen. 2019;58(4):500-10.

22. Chen R, Zhu S, Fan X-G, Wang H, Lotze MT, Zeh HJ, Billiar TR, Kang R, Tang D. High mobility group protein B1 controls liver cancer initiation through yes-associated protein -dependent aerobic glycolysis. Hepatology (Baltimore, MD). 2018;67(5):1823-41.

23. Yang J, Zhang W. New molecular insights into osteosarcoma targeted therapy. Curr Opin Oncol. 2013;25(4):398-406.

24. Kang H-M, Park B-S, Kang H-K, Park H-R, Yu S-B, Kim I-R. Delphinidin induces apoptosis and inhibits epithelial-to-mesenchymal transition via the ERK/p38 MAPK-signaling pathway in human osteosarcoma cell lines. Environ Toxicol. 2018;33(6):640-9.

25. Vo JN, Cieslik M, Zhang Y, Shukla S, Xiao L, Zhang Y, Wu Y-M, Dhanasekaran SM, Engelke CG, Cao X, et al. The landscape of circular RNA in cancer. Cell. 2019;176(4):869-81.

26. Zhang H-d, Jiang L-H, Sun D-W, Hou J-C, Ji Z-L. CircRNA: a novel type of biomarker for cancer. Breast Cancer. 2018;25(1):1-7.

27. Jin J, Chen A, Qiu W, Chen Y, Li Q, Zhou X, Jin D. Dysregulated cirCRNA_100876 suppresses proliferation of osteosarcoma cancer cells by targeting microRNA-136. J Cell Biochem. 2019;120(9):15678-87.

28. Wu Y, Xie Z, Chen J, Chen J, Ni W, Ma Y, Huang K, Wang G, Wang J, Ma J, et al. Circular RNA circTADA2A promotes osteosarcoma progression and metastasis by sponging miR-203a-3p and regulating CREB3 expression. Mol Cancer. 2019:18(1):73.

29. Li S, Pei Y, Wang W, Liu F, Zheng K, Zhang X. Circular RNA 0001785 regulates the pathogenesis of osteosarcoma as a ceRNA by sponging miR-1200 to upregulate HOXB2. Cell Cycle (Georgetown, Tex). 2019;18(11):1281-91.

30. Liu K, Huang J, Ni J, Song D, Ding M, Wang J, Huang X, Li W. MALAT1 promotes osteosarcoma development by regulation of HMGB1 via miR142-3p and miR-129-5p. Cell Cycle. 2017;16(6):578-87.

31. Liu Y-J, Li W, Chang F, Liu J-N, Lin J-X, Chen D-X. MicroRNA-505 is downregulated in human osteosarcoma and regulates cell proliferation, migration and invasion. Oncol Rep. 2018;39(2):491-500.

32. Huang J, Liu K, Yu Y, Xie M, Kang R, Vernon P, Cao L, Tang D, Ni J. Targeting HMGB1-mediated autophagy as a novel therapeutic strategy for osteosarcoma. Autophagy. 2012;8(2):275-7.

\section{Publisher's Note}

Springer Nature remains neutral with regard to jurisdictional claims in published maps and institutional affiliations.

Ready to submit your research? Choose BMC and benefit from:

- fast, convenient online submission

- thorough peer review by experienced researchers in your field

- rapid publication on acceptance

- support for research data, including large and complex data types

- gold Open Access which fosters wider collaboration and increased citations

- maximum visibility for your research: over $100 \mathrm{M}$ website views per year

At BMC, research is always in progress.

Learn more biomedcentral.com/submissions 